\title{
Non-parametric Sequential Estimation of a Regression Function Based on Dependent Observations
}

\author{
Dimitris N. Politis and Vyacheslav A. Vasiliev \\ Department of Mathematics, University of California, San Diego, California, USA \\ Department of Applied Mathematics and Cybernetics, Tomsk State University, Tomsk, Russia
}

\begin{abstract}
This paper presents a sequential estimation procedure for an unknown regression function. Observed regressors and noises of the model are supposed to be dependent and form sequences of dependent numbers. Two types of estimators are considered. Both estimators are constructed on the basis of Nadaraya-Watson kernel estimators.

First, sequential estimators with given bias and mean square error are defined. According to the sequential approach the duration of observations is a special stopping time. Then on the basis of these estimators of a regression function, truncated sequential estimators on a time interval of a fixed length are constructed. At the same time the variance of these estimators is controlled by a (non-asymptotic) bound.

In addition to non-asymptotic properties, the limiting behavior of presented estimators is investigated. It is shown, in particular, that by the appropriate chosen bandwidths both estimators have optimal (as compared to the case of independent data) rates of convergence of Nadaraya-Watson kernel estimators.
\end{abstract}

Keywords: Non-parametric kernel regression estimation; Dependent observations; Sequential approach; Guaranteed mean square accuracy; Finite sample size.

Subject Classifications: 62G05; 62G08; 62G20; 62J02; 62L12.

\section{INTRODUCTION}

This paper considers the problem of non-parametric kernel estimation of a regression function $f(x), x \in \mathbb{R}^{1}$ at a point $x=x^{0}$ satisfying the equation

$$
Y_{i}=f\left(x_{i}\right)+\delta_{i}, \quad i \geq 1,
$$

in the case of dependent regressors $X=\left(x_{i}\right)_{i \geq 1}$ and noises $\Delta=\left(\delta_{i}\right)_{i \geq 1}$. It is supposed, that pairs $\left(Y_{i}, x_{i}\right)$, $i=1,2, \ldots$ are under observations but the errors $\delta_{i}$ are unobservable. We allow in the paper for general dependence conditions on random variables $\left(x_{i}\right)$ and $\left(\delta_{i}\right)$ supposing mutual dependence of the processes $X$ and $\Delta$. In particular, model inputs can be unbounded with a positive probability and estimation problem of non-linear autoregressive function can be solved (see Section 5.2).

There are a lot of results and publications dedicate this problem for independent and dependent observations, in asymptotic and non-asymptotic problem statements (see, e.g, Bai and Liu (2007), Dharmasena at al. (2008)-Hondaa (1998), Leonov (1999), Nazin and Ljung (2002), Politis at al. (1999)-Scott (1992) etc.) It should be noted, that often regressors $X$ a supposed to be non-random or bounded and noises $\Delta$ of the model are independent, see Bai and Liu (2007); Dharmasena at al. (2008); Györfi at al. (2002); Nazin and Ljung (2002); Politis at al. (1999), Roll at al. (2002)-Rosenblatt (1991) etc.

Address correspondence to V. A. Vasiliev, Department of Applied Mathematics and Cybernetics, Tomsk State University, Lenin Ave. 36, Tomsk, 634050, Russia; Fax: (382) 252-9895; Email: vas@ mail.tsu.ru 
The main goal of the paper is twofold. We will construct sequential and so-called truncated sequential estimators of a regression function. Estimators of both types have known mean square errors (MSE's). To obtain sequential estimators with an arbitrary accuracy one needs to have a sample of unbounded size. This approach has been primarily proposed by A.Wald for various statistical problems for a scheme of independent observations, see Wald $(1947,1950)$. It proposes a construction of a special stopping time as a duration of observations to obtain statistics with desired properties. Then this idea has been applied to parameter estimation problem of one-parameter stochastic differential equations in Liptser and Shiryaev (1978); Novikov (1971) and for multiparameter continuous and discrete-time dynamic systems in many papers and books (see Borisov and Konev (1977); Dobrovidov at al. (2012); Konev (1985); Küchler and Vasiliev (2010) among others). Sequential approach has also been applied to non-parametric regression and density function estimation problems as well (see, e.g., Carroll (1976); Davies and Wegman (1975), Dharmasena at al. (2008)-Efroimovich (2007), Hondaa (1998); Liski at al. (2002); Martinsek (1992); Mukhopadhyay and Solanky (1994); Rao (1983); Stute (1983); Vasiliev (1997); Vasiliev and Koshkin (1998); Xu and Martinsek (1995)). Peculiarities and difficulties of sequential approach to nonparametric problems are discussed in detail in Efroimovich (2007).

However in practice the observation time of a system is usually not only finite but fixed which does not allow for the use of such estimators. One of the possibilities for finding estimators with the guaranteed quality of inference using a sample of fixed size is provided by the approach of truncated sequential estimation. Truncated sequential estimation method was developed in Konev and Pergamenshchikov (1990a)-Konev and Pergamenshchikov (1992), as well as in Fourdrinier at al. (2009) for parameter estimation problems in discrete and continuous time dynamic models. Using sequential approach, they have constructed estimators of dynamic systems parameters with known variance by samples of fixed size.

Non-parametric truncated sequential estimators of a regression function presented in this paper constitute Nadaraya-Watson estimators calculated at a special stopping time. These estimators have known mean square errors as well. The duration of observations is also random but bounded from above to a non-random fixed number.

Non-asymptotic and asymptotic properties of estimators of both types are investigated. It is shown, that truncated sequential estimators coincide with the afore-mentioned sequential estimators (i.e. with the Nadaraya-Watson estimators calculated at a special stopping time) for sufficiently large sample size.

It should be noted, that usually non-parametric estimators of functionals are studied by asymptotic assumptions even in sequential problem statement (except of some special cases, see, e.g., Dobrovidov at al. (2012) and references therein). Nadaraya-Watson estimators of a regression function with given nonasymptotic properties are derived here for the first time.

The outline of the paper is as follows. In Section 2 basic assumptions on a regression function to be estimated, as well as regressors and noises of the model are given. In Section 3 we present a construction and basic properties of sequential estimation procedure of the regression function, which can be estimated with an arbitrary mean square accuracy. Section 4 presents truncated sequential estimation procedure, which give a possibility to obtain an estimator of the regression function with a known variance based on a sample of finite size. In Section 5 we consider basic examples of dependence type of regressors and noises of the model. In particular, non-linear autoregressive estimation problem is considered. Section 6 is the conclusion of the paper. In Section 7 all proves are given.

Similar results were presented in Politis and Vasiliev (2012).

\section{PROBLEM SET-UP AND BASIC ASSUMPTIONS}

Consider the problem of non-parametric estimation of a regression function $f(x)$ at a point $x=x^{0}$ from the equation (1.1) based on data $\left(Y_{i}, x_{i}\right), i=1,2, \ldots$

The notation $\overline{1, m}$ means the set of integers $1,2, \ldots, m$. 
We suppose that the function to be estimated satisfies the following assumption.

Assumption (f): The function $f(x)$ is $p$-th differentiable at a point $x^{0}, p \geq 0$ and for some positive numbers $C_{f, i}, i=\overline{0, p}(p \geq 0), L_{f}, \gamma_{f} \in(0,1]$, such that

$$
\left|f^{(i)}\left(x^{0}\right)\right| \leq C_{f, i}, i=\overline{0, p}
$$

and for every $x, y \in \mathbb{R}^{1}$,

$$
\left|f^{(p)}(x)-f^{(p)}(y)\right| \leq L_{f}|x-y|^{\gamma_{f}}
$$

For estimation of the function $f(\cdot)$ at a point $x^{0}, f=f\left(x^{0}\right)$ we use so-called sequential estimators, constructed on the basis of kernel type estimators of the form

$$
\hat{f}_{N}=\left(\sum_{i=1}^{N} K\left(\frac{x^{0}-x_{i}}{h}\right)\right)^{+} \cdot \sum_{i=1}^{N} Y_{i} K\left(\frac{x^{0}-x_{i}}{h}\right), \quad N \geq 1
$$

where $a^{+}=a^{-1}$ for $a \neq 0$; and $a^{+}=0$ when $a=0 ; K(\cdot)$ is a kernel function and $h$ is a bandwidth, satisfying the Assumptions (K) and (h) below respectively.

Define for every $i \geq 1$ density functions $f_{i-1}(t)$ of conditional distribution functions $F_{i-1}(t)=P\left(x_{i} \leq\right.$ $\left.t \mid \mathcal{F}_{i-1}^{x}\right), \mathcal{F}_{i}^{x}=\sigma\left\{x_{1}, \ldots, x_{i}\right\}$.

As regards to the regressors $X$ and $\Delta$ we suppose

Assumption (X): Assume the conditional density functions $f_{i-1}(t), i \geq 1$ are $q$-th differentiable at a point $x^{0}, q \geq 0$ and for some positive numbers $c_{x}^{0}, C_{x, k}, k=\overline{0, q}(q \geq 0), L_{x}$ and $\gamma_{x} \in(0,1]$ the following relations

$$
\begin{gathered}
\inf _{i \geq 1} f_{i-1}\left(x^{0}\right) \geq c_{x}^{0}, \\
\sup _{i \geq 1}\left|f_{i-1}^{(k)}\left(x^{0}\right)\right| \leq C_{x, k}, k=\overline{0, q}
\end{gathered}
$$

and for every $x, y \in \mathbb{R}^{1}$,

$$
\sup _{i \geq 1}\left|f_{i-1}^{(q)}(x)-f_{i-1}^{(q)}(y)\right| \leq L_{x}|x-y|^{\gamma_{x}}
$$

are fulfilled.

Assumption $(\boldsymbol{\Delta})$ : Let the noises $\Delta$ be conditionally zero mean $E\left(\delta_{i} \mid \mathcal{F}_{i}^{x}\right)=0, i \geq 1$ and for some monotonically non-increasing as $k \rightarrow \infty$ non-negative function $\varphi(k), k \geq 1$ the following "general mixing condition" for the sequence $\Delta$ holds true

$$
\left|E\left(\delta_{i} \delta_{j} \mid \mathcal{F}_{i \vee j}^{x}\right)\right| \leq \varphi(|i-j|), \quad i, j \geq 1 .
$$

Define admissible sets of kernels $K(\cdot)$ and bandwidths $h$ as follows.

Assumption (K): Let the function $K(\cdot)$ is uniformly bounded from above:

$$
\sup _{z}|K(z)| \leq \bar{K}<\infty
$$


and such that

$$
\int K(z) d z=1, \quad \int\left|z^{s} K(z)\right| d z<\infty, \quad 0 \leq s \leq q+\gamma_{x}+2\left(p+\gamma_{f}\right)
$$

Moreover, we suppose that the integrals

$$
\int z^{j} K(z) d z=0, \quad j=\overline{1, p+q}
$$

Assumption (h): Let $\left(h_{n}\right)_{n \geq 1}$ be a sequence of monotonically decreasing to zero positive numbers such that $n h_{n} \rightarrow \infty$ as $n \rightarrow \infty$ and $h_{1} \leq 1$.

It should be noted that, according to Assumption (K), the allowed kernels can be of order higher than two, i.e. not necessarily nonnegative everywhere. For example, the infinite-order flat-top kernels of Politis (2001) are allowed, including the infinitely differentiable flat-top kernel, see McMurry and Politis (2004).

\section{ESTIMATION OF THE REGRESSION FUNCTION WITH GUARANTEED ACCURACY}

In this section we consider sequential estimators of the regression function $f$ at a point $x^{0}$ with a given variance, calculated at a special stopping time. These estimators are constructed on the basis of NadarayaWatson kernel estimators. Bandwidth giving an optimal convergence rate of estimators (which coincides with the rate of Nadaraya-Watson estimators constructed by independent observations) is found.

\subsection{Sequential Estimation Procedure}

According to sequential approach we introduce the following definition.

Definition 3.1. Let $\left(H_{n}\right)_{n \geq 1}$ be an unboundedly monotonically increasing given sequence of positive numbers and $\left(h_{n}\right)_{n \geq 1}$ satisfies Assumption $(h)$.

The sequential plans $\left(\tau_{n}, f_{n}^{*}\right), n \geq 1$ of estimation of the function $f=f\left(x^{0}\right)$ will be defined by the formulae

$$
\begin{gathered}
\tau_{n}=\inf \left\{j \geq 1: \sum_{i=1}^{j} K\left(\frac{x^{0}-x_{i}}{h_{n}}\right) \geq H_{n}\right\}, \\
f_{n}^{*}=\frac{1}{Q_{n}} \sum_{i=1}^{\tau_{n}} Y_{i} K\left(\frac{x^{0}-x_{i}}{h_{n}}\right), \quad Q_{n}=\sum_{i=1}^{\tau_{n}} K\left(\frac{x^{0}-x_{i}}{h_{n}}\right),
\end{gathered}
$$

where $\tau_{n}$ is the duration of estimation, and $f_{n}^{*}$ is the estimator of $f$ with given accuracy in the mean square sense.

We have introduced in Definition 3.1 the sequence of sequential estimation plans. At the same time Theorem 3.1 below gives non-asymptotic properties of these plans for every $n \geq 1$, in particular, upper bound for the MSE of the estimator $f_{n}^{*}$. It gives a possibility to investigate asymptotic properties of defined plans as $n \rightarrow \infty$ as well (see Section 3.2).

To formulate this theorem we need the following notation.

Assume $\left(l_{n}\right)_{n \geq 1}$ to be an arbitrary non-decreasing sequence of positive numbers. 
Define the function $\gamma_{x, \delta}=0$ if processes $X$ and $\Delta$ are independent and 1 otherwise; $\gamma_{\delta}=0$ if $\varphi(k)=0$, $k>0$ and 1 otherwise; the numbers

$$
\begin{gathered}
\varphi_{n}=\varphi\left(l_{n}\right), \quad K_{\alpha}=\int\left|z^{\alpha} K(z)\right| d z, \quad \alpha \geq 0, \quad \kappa=(q !)^{-1} L_{x} K_{q+\gamma_{x}}, \\
C_{1}=\frac{1}{q !} \sum_{j=1}^{p} \frac{1}{j !} C_{f, j} K_{q+j+\gamma_{x}} \chi(p>0), \quad C_{2}=\frac{1}{p !} C_{x, 0} K_{p+\gamma_{f}}, \quad C_{3}=C_{x, 0} \int K^{2}(y) d y, \\
C_{4}=4 \bar{K}(p+2) \sum_{j=0}^{q+1} \sum_{k=0}^{p+1} \frac{1}{j !(k !)^{2}} C_{x, j} C_{f, k}^{2} K_{\beta(j, k)}, \quad C_{x, q+1}=\frac{1}{q !} L_{x}, \quad C_{f, p+1}=\frac{1}{p !} L_{f}, \\
\beta(j, k)=j+2 k+\left(\gamma_{x}-1\right) \chi(j=q+1)+2\left(\gamma_{f}-1\right) \chi(k=p+1),
\end{gathered}
$$

as well as

$$
\begin{gathered}
n_{0}=\inf \left\{n \geq 1: c_{x}^{0}-\kappa h_{n}^{q+\gamma_{x}}>0\right\} \\
t_{n}=\frac{\left(C_{x, 0}+\kappa h_{n}^{q+\gamma_{x}}\right) \cdot \sqrt{C_{3}\left(H_{n}+\bar{K}\right)}}{\left(c_{x}^{0}-\kappa h_{n}^{q+\gamma_{x}}\right)^{5 / 2} h_{n}} \\
+\frac{\sqrt{\left[C_{3}\left(C_{x, 0}+\kappa h_{n}^{q+\gamma_{x}}\right)^{2} \cdot\left(c_{x}^{0}-\kappa h_{n}^{q+\gamma_{x}}\right)^{-3}+2 \bar{K}^{2}\left(c_{x}^{0}-\kappa h_{n}^{q+\gamma_{x}}\right)^{-1}\right]\left(H_{n}+\bar{K}\right)+H_{n}^{2}}}{\left(c_{x}^{0}-\kappa h_{n}^{q+\gamma_{x}}\right) h_{n}} \\
w_{n}=\sqrt{\varphi(0) \bar{K}^{2} \gamma_{\delta} \frac{l_{n}}{H_{n}^{2}}\left(1+3 l_{n}\right)+C_{3} \varphi(0)\left(c_{x}^{0}-\kappa h_{n}^{q+\gamma_{x}}\right)^{-1} \frac{1}{H_{n}}\left(1+\frac{\bar{K}}{H_{n}}\right)} \\
+2 \gamma_{\delta} \varphi(0) K_{0} \bar{K}\left(c_{x}^{0}-\kappa h_{n}^{q+\gamma_{x}}\right)^{-1} \frac{l_{n}}{H_{n}}\left(1+\frac{\bar{K}}{H_{n}}\right)+2 \gamma_{\delta} K_{0} \bar{K} \varphi_{n} \frac{h_{n} t_{n}^{2}}{H_{n}^{2}} \\
b_{n}=\left(c_{x}^{0}-\kappa h_{n}^{q+\gamma_{x}}\right)^{-1}\left[C_{1} h_{n}^{q+1+\gamma_{x}}+C_{2} h_{n}^{p+\gamma_{f}}\right]\left(1+\frac{\bar{K}}{H_{n}}\right)+w_{n}
\end{gathered}
$$

and

$$
\begin{aligned}
V_{n}=2 & \left(2 C_{3} C_{f, 0}^{2}+\left(1+\gamma_{x, \delta}\right) C_{4}\right)\left(c_{x}^{0}-\kappa h_{n}^{q+\gamma_{x}}\right)^{-1} \frac{1}{H_{n}}\left(1+\frac{\bar{K}}{H_{n}}\right) \\
& \left.+\left(1+\gamma_{x, \delta}\right) w_{n}^{2}+\frac{2\left(C_{1} h_{n}^{q+1+\gamma_{x}}+C_{2} h_{n}^{p+\gamma_{f}}\right)^{2} h_{n}^{2} t_{n}^{2}}{H_{n}^{2}}\right\} .
\end{aligned}
$$

Theorem 3.1. Assume model (1.1) and let the Assumptions (f), $(K),(X),(h)$ and $(\Delta)$ be fulfilled and $n \geq n_{0}$. Then the sequential estimation plans $\left(\tau_{n}, f_{n}^{*}\right)$ are closed, i.e. $\tau_{n}<\infty$ a.s. and have the following nonasymptotic properties:

1. for the expected duration of observations

a)

$$
\left(C_{x, 0}+\kappa h_{n}^{q+\gamma_{x}}\right)^{-1} h_{n}^{-1} H_{n} \leq E \tau_{n} \leq\left(c_{x}^{0}-\kappa h_{n}^{q+\gamma_{x}}\right)^{-1} h_{n}^{-1}\left(H_{n}+\bar{K}\right),
$$

b)

$$
E \tau_{n}^{2} \leq t_{n}^{2}
$$

2. for the bias

$$
\left|E f_{n}^{*}-f\right| \leq b_{n}
$$

3. for the MSE

$$
E\left(f_{n}^{*}-f\right)^{2} \leq V_{n}
$$


Proof of Theorem 3.1 is given in the Appendix.

Remark 3.1. From Definition 3.1 it follows that the presented sequential estimators coincide with the usual Nadaraya-Watson estimators calculated at a special stopping time. Therefore, at least in the case of independent inputs of the model, these estimators have the same asymptotic properties as Nadaraya-Watson estimators. However, sequential estimators have the above exact, non-asymptotic properties, that may be important for practitioners.

\subsection{Bandwidth Choice for Sequential Estimators}

From Theorem 3.1 follows, that it is natural to define $H_{n}=n h_{n}$. In this case and under the condition on the bandwidth $n h_{n} \rightarrow \infty$ as $n \rightarrow \infty$ from Assumption (h) the expected duration of observations is proportional to $n$, i.e.,

$$
C_{x, 0}^{-1} n-\frac{C_{x, 0}^{-1} \kappa h_{n}^{q+\gamma_{x}} n}{C_{x, 0}+\kappa h_{n}^{q+\gamma_{x}}} \leq E \tau_{n} \leq\left(c_{x}^{0}\right)^{-1} n+\frac{\left(c_{x}^{0}\right)^{-1} \kappa h_{n}^{q+\gamma_{x}} n+\bar{K} h_{n}^{-1}}{c_{x}^{0}-\kappa h_{n}^{q+\gamma_{x}}}, \quad n \geq n_{0},
$$

as well as

$$
E \tau_{n}^{2} \leq\left(c_{x}^{0}\right)^{-2} n^{2}+o\left(n^{2}\right) \quad \text { as } \quad n \rightarrow \infty
$$

and

$$
\begin{gathered}
\left|E f_{n}^{*}-f\right|=O\left(h_{n}^{q+1+\gamma_{x}}+h_{n}^{p+\gamma_{f}}+\sqrt{\varphi_{n} h_{n}^{-1}}+\frac{1+\gamma_{\delta} \sqrt{l_{n}}}{\sqrt{n h_{n}}}\right), \\
E\left(f_{n}^{*}-f\right)^{2}=O\left(h_{n}^{2\left(q+1+\gamma_{x}\right)}+h_{n}^{2\left(p+\gamma_{f}\right)}+\varphi_{n} h_{n}^{-1}+\frac{1+\gamma_{\delta} l_{n}}{n h_{n}}\right) \quad \text { as } n \rightarrow \infty .
\end{gathered}
$$

From these formulae it follows, that for the asymptotic unbiasnessy and $L_{2}$-convergency of $f_{n}^{*}$ we have to use the sequences $\left(l_{n}\right)$ and $\left(h_{n}\right)$ satisfying the condition

$$
\varphi_{n} h_{n}^{-1}+\frac{l_{n}}{n h_{n}}=o(1) \quad \text { as } n \rightarrow \infty .
$$

Remark 3.2. If the regressors $x_{i}$ form a sequence of i.i.d.r.v's, then $c_{x}^{0}=C_{x, 0}$. Moreover if the number $c_{x}^{0}=f_{i}\left(x^{0}\right)$ is known, we can put $H_{n}=c_{x}^{0} n h_{n}$. In this case

$$
n-\frac{\kappa h_{n}^{q+\gamma_{x}} n}{c_{x}^{0}+\kappa h_{n}^{q+\gamma_{x}}} \leq E \tau_{n} \leq n+\frac{\kappa h_{n}^{q+\gamma_{x}} n+\bar{K} h_{n}^{-1}}{c_{x}^{0}-\kappa h_{n}^{q+\gamma_{x}}}
$$

and

$$
\varlimsup_{n \rightarrow \infty}\left[\left(h_{n}^{q+\gamma_{x}} n\right)^{-1} \wedge h_{n}\right]\left|E \tau_{n}-n\right|<\infty,
$$

where $a \wedge b=\min \{a, b\}$, as well as

$$
E \tau_{n}^{2} \leq n^{2}+o\left(n^{2}\right) \text { as } n \rightarrow \infty .
$$

It should be noted that in the case of i.i.d. noises $\left(\delta_{i}\right)$ the MSE of the constructed sequential estimator has similar optimal decreasing rate

$$
E\left(f_{n}^{*}-f\right)^{2}=O\left(h_{n}^{2\left(q+1+\gamma_{x}\right)}+h_{n}^{2\left(p+\gamma_{f}\right)}+\frac{1}{n h_{n}}\right) \quad \text { as } n \rightarrow \infty
$$

as in the case of i.i.d. regressors $\left(x_{i}\right)$.

In particular, for the case $p=0, \gamma_{f}=1$ (see, for comparison, Györfi at al. (2002) among others),

$$
E\left(f_{n}^{*}-f\right)^{2}=O\left(h_{n}^{2}+\frac{1}{n h_{n}}\right) \quad \text { as } n \rightarrow \infty .
$$




\section{TRUNCATED SEQUENTIAL ESTIMATION OF THE REGRESSION FUNCTION $f$}

We shall consider in this section the problem of estimation of the regression function $f$ with a known mean square accuracy based on observations of the process $\left(Y_{i}, x_{i}\right)$ for $i=1, \ldots, N$ on the time interval $[1, N]$ for a fixed time $N$. These estimators are constructed on the basis of sequential estimators considered in the previous section.

Such possibility gives the so-called truncated sequential estimation method, developed in Konev and Pergamenshchikov (1990a)-Konev and Pergamenshchikov (1992), as well as in Fourdrinier at al. (2009) for parameter estimation problems in discrete and continuous time dynamic models.

Bandwidth giving an optimal convergence rate of estimators is found.

\subsection{Truncated Sequential Estimation Procedure}

Definition 4.1. Let $H$ and $h$ be positive numbers.

The truncated sequential plans $\left(\tau_{N}(h, H), f_{N}^{*}(h, H)\right), N \geq 1$ of estimation of the function $f=f\left(x^{0}\right)$ will be defined by the formulae

$$
\tau_{N}(h, H)=\inf \left\{j \in[1, N]: \sum_{i=1}^{j} K\left(\frac{x^{0}-x_{i}}{h}\right) \geq H\right\}
$$

with the provision that $\inf \{\varnothing\}=N$,

$$
\begin{gathered}
f_{N}^{*}(h, H)=\hat{f}_{N}(h, H) \cdot \chi\left(\sum_{i=1}^{N} K\left(\frac{x^{0}-x_{i}}{h}\right) \geq H\right)+\frac{C_{f, 0}}{2} \cdot \chi\left(\sum_{i=1}^{N} K\left(\frac{x^{0}-x_{i}}{h}\right)<H\right), \\
\hat{f}_{N}(h, H)=\frac{1}{Q_{N}(h, H)} \sum_{i=1}^{\tau_{N}(h, H)} Y_{i} K\left(\frac{x^{0}-x_{i}}{h}\right), \quad Q_{N}(h, H)=\sum_{i=1}^{\tau_{N}(h, H)} K\left(\frac{x^{0}-x_{i}}{h}\right),
\end{gathered}
$$

where $\tau_{N}(h, H)$ is the duration of estimation, which is bounded by construction $\left(\tau_{N}(h, H) \leq N\right)$ and $f_{N}^{*}(h, H)$ is the estimator of $f$. The notation $\chi(A)$ means the indicator function of set $A$.

The parameters $H$ and $h$ will be defined in a special way as functions of $N$ for optimization of a convergence rate of the estimator $f_{N}^{*}(h, H)$ as $N \rightarrow \infty$ in the mean square sense.

Assume $l(H)$ to be an arbitrary non-decreasing function of positive numbers.

Define

$$
\begin{aligned}
V_{N}(h, H)= & 2\left\{\left(2 C_{3} C_{f, 0}^{2}+\left(1+\gamma_{x, \delta}\right) C_{4}\right) \frac{h N}{H^{2}}+\left(1+\gamma_{x, \delta}\right)\left[\varphi(0) \bar{K}^{2} \gamma_{\delta} \frac{l(H)}{H^{2}}(1+3 l(H))\right.\right. \\
& \left.+C_{3} \varphi(0) \frac{h N}{H^{2}}+2 \gamma_{\delta} \varphi(0) K_{0} \frac{l(H) h N}{H^{2}}+2 \gamma_{\delta} K_{0} \bar{K} \varphi(l(H)) \frac{h N^{2}}{H^{2}}\right] \\
+ & \left.\frac{2\left(C_{1} h^{q+1+\gamma_{x}}+C_{2} h^{p+\gamma_{f}}\right)^{2} h^{2} N^{2}}{H^{2}}\right\}+\frac{C_{3} C_{f, 0}^{2} N h}{4\left[N h\left(c_{x}^{0}-\kappa h^{q+\gamma_{x}}\right)-H\right]^{2}} .
\end{aligned}
$$

From the proof of the following Theorem 4.1 it follows that the number $H$ satisfies the condition

$$
N h\left(c_{x}^{0}-\kappa h^{q+\gamma_{x}}\right)-H>0 .
$$

Thus it is natural to put

$$
H:=H_{N}=N h_{N} C_{\alpha}^{-1},
$$


where

$$
C_{\alpha}=\left(c_{x}^{0}-\kappa h_{n_{0}}^{q+\gamma_{x}}-\alpha^{-1 / 2}\right)^{-1}, \quad \alpha>\left(c_{x}^{0}-\kappa h_{n_{0}}^{q+\gamma_{x}}\right)^{-2}
$$

and $\left(h_{N}\right)$ is a sequence satisfying Assumption (h). It should be noted that, according to the assumptions of the theorem below the number $C_{\alpha}$ and the sequence $\left(H_{N}\right)$ are assumed known.

Then, denoting $l_{N}=l\left(H_{N}\right)$ and $\varphi_{N}=\varphi\left(l_{N}\right)$, we have

$$
\begin{gathered}
V_{N}^{0}:=V_{N}\left(h_{N}, H_{N}\right)=2\left\{\left[\left(2 C_{3} C_{f, 0}^{2}+\left(1+\gamma_{x, \delta}\right) C_{4}\right) C_{\alpha}^{2}+\frac{C_{3} C_{f, 0}^{2} \alpha}{8}+\left(1+\gamma_{x, \delta}\right)\right.\right. \\
\left.\times\left(C_{3} C_{\alpha}^{2} \varphi(0)+2 \gamma_{\delta} \varphi(0) K_{0} C_{\alpha}^{2} l_{N}\right)\right] \frac{1}{N h_{N}}+\left(1+\gamma_{x, \delta}\right)\left[\varphi(0) \bar{K}^{2} C_{\alpha}^{2} \gamma_{\delta} \frac{l_{N}}{\left(N h_{N}\right)^{2}}\left(1+3 l_{N}\right)\right. \\
\left.\left.+2 \gamma_{\delta} K_{0} \bar{K} C_{\alpha}^{2} \varphi_{N} h_{N}^{-1}\right]+2 C_{\alpha}^{2}\left(C_{1} h_{N}^{q+1+\gamma_{x}}+C_{2} h_{N}^{p+\gamma_{f}}\right)^{2}\right\} .
\end{gathered}
$$

The main result of this section is the following

Theorem 4.1. Assume model (1.1) and let the Assumptions (f), $(K),(X)$ and $(\Delta)$ be fulfilled, where the number $C_{f, 0}$ in Assumption ( $f$ ) is supposed to be known. Then:

1) for every positive numbers $h, H$ satisfying the condition (4.2) the truncated sequential estimator $f_{N}^{*}(h, H)$ has the MSE:

$$
E\left(f_{N}^{*}(h, H)-f\right)^{2} \leq V_{N}(h, H)
$$

2) for $H=\left(H_{N}\right)$ defined in (4.3) and $\left(h_{N}\right)$ satisfying (4.2) it holds

$$
E\left(f_{N}^{*}\left(h_{N}, H_{N}\right)-f\right)^{2} \leq V_{N}^{0}
$$

3) assume there exists a number $r>2$, such that the bandwidth $h=\left(h_{N}\right)$ from Assumption $(h)$ satisfies the additional condition

$$
\sum_{N \geq 1} \frac{1}{h_{N}^{r-1} N^{r / 2}}<\infty
$$

and $H=\left(H_{N}\right)$ is defined in (4.3). Then

$$
\lim _{N \rightarrow \infty} \frac{\tau_{N}\left(h_{N}, H_{N}\right)}{N}<1 \quad \text { a.s. }
$$

and the estimator $f_{N}^{*}\left(h_{N}, H_{N}\right)$ is non-degenerate as $N \rightarrow \infty$ in the following sense:

$$
P\left(f_{N}^{*}\left(h_{N}, H_{N}\right)=\hat{f}_{N}\left(h_{N}, H_{N}\right)\right)=1 \text { for } N \text { large enough. }
$$

Proof of Theorem 4.1 is given in the Appendix.

Remark 4.1. From the third assertion of Theorem 4.1 it follows, that similarly to sequential estimators, the truncated sequential estimators have the asymptotic properties of Nadaraya-Watson estimators (2.1). However, in distinction from the sequential estimators considered in Section 3.1, the truncated estimators have known variance for fixed (non-random) sample sizes.

Remark 4.2. If the number $C_{f, 0}$ in Assumption $(f)$ is unknown, then the Definition 2.2 of sequential estimator is modified as follows:

$$
f_{N}^{*}(h, H)=\hat{f}_{N}(h, H) \cdot \chi\left(\sum_{i=1}^{N} K\left(\frac{x^{0}-x_{i}}{h}\right) \geq H\right) .
$$

All assertions of Theorem 4.1 will be fulfilled with slightly changed (but having similar structure) functions $V_{N}(h, H)$ and $V_{N}^{0}$. More precisely, the number 4 in the denominator of the last summand in the definition of $V_{N}(h, H)$ should be omitted and the number 8 in the denominator of the third summand in the right hand side in the definition of $V_{N}^{0}$ should be changed to the number 2. 


\subsection{Bandwidth Choice for Truncated Sequential Estimators}

From Theorem 4.1 it follows, that by the definition of $V_{N}^{0}$ and assertion 2) of Theorem 4.1, the truncated sequential estimator (4.1) has similar to the sequential estimator (3.1) rate of convergency (as $N \rightarrow \infty$ ).

In the case of i.i.d. noises $\left(\delta_{i}\right)$ the MSE of the constructed sequential estimator has optimal decreasing rate

$$
E\left(f_{N}^{*}\left(h_{N}, H_{N}\right)-f\right)^{2}=O\left(h_{N}^{2 \varrho}+\frac{1}{N h_{N}}\right) \quad \text { as } N \rightarrow \infty,
$$

$\varrho=\left(q+1+\gamma_{x}\right) \wedge\left(p+\gamma_{f}\right)$.

Then the bandwidth $h$ with the optimal rate is proportional to

$$
h_{N} \sim N^{-\frac{1}{(2 \varrho+1)}}
$$

and the condition (4.4) is fulfilled for $\varrho>1 / 2$ and

$$
r>\frac{4 \varrho}{2 \varrho-1} .
$$

In particular, for the case $p=0, \gamma_{f}=1$,

$$
E\left(f_{N}^{*}\left(h_{N}, H_{N}\right)-f\right)^{2}=O\left(h_{N}^{2}+\frac{1}{N h_{N}}\right) \quad \text { and } r>4 .
$$

Remark 4.3. Theorems 3.1 and 4.1 give known upper bounds for the bias and the MSE of presented estimators as well as for the mean of observation time in Theorem 3.1 if the parameters of classes of functions, introduced in Assumptions $(f)$ and $(X)$ are known $\left(C_{f, k}, p, \gamma_{f}\right.$, etc $)$. In this case 'optimal' bandwidth can be found from minimization of the upper bounds for the MSE's.

At the same time the assertions of Theorems 3.1 and 4.1 are fulfilled even if some of these parameters are unknown. Estimators with such properties can be successfully used in various adaptive procedures as pilot estimators (see, e.g., Dobrovidov at al. (2012), Vasiliev (1997)-Vasiliev and Koshkin (1998)).

\section{EXAMPLES}

In this section we consider examples of dependence types of observed regressors and model noises satisfying Assumptions $(\mathrm{X})$ and $(\Delta)$.

\subsection{Case of Independent Regressors $X$ and $\Delta$}

Consider examples of inputs of the model in this case.

\subsubsection{Example of Regressors $X$}

Assume that regressors $\left(x_{i}\right)$ satisfy the following equation

$$
x_{i}=\Psi\left(x_{i-1}, \ldots, x_{i-r}\right)+\varepsilon_{i}, \quad i \geq 1,
$$

where $\Psi(\cdot)$ is a bounded function $|\Psi(y)| \leq \bar{\Psi}<\infty, y \in \mathcal{R}^{r}, r \geq 1$.

Assume that the input noises $\varepsilon_{i}$ in the model (5.1) are i.i.d. with the density function $f_{\varepsilon}(\cdot)$ and independent from the initial vector $\left(x_{0}, x_{-1}, \ldots, x_{1-r}\right)$. In this case $f_{i-1}(t)=f_{\varepsilon}\left(t-\Psi\left(x_{i-1}, \ldots, x_{i-r}\right) \mid \mathcal{F}_{i-1}\right)$. 
Thus Assumption (X) holds true if the density function $f_{\varepsilon}(\cdot)$ is $q$-th differentiable, $q \geq 0$ and for some positive numbers $c_{x}^{0}, C_{x, 0}, L_{x}$ and $\gamma_{x} \in(0,1]$ the following relations

$$
\begin{gathered}
\inf _{\left|t-x^{0}\right| \leq \bar{\Psi}} f_{\varepsilon}(t) \geq c_{x}^{0}, \\
\sup _{\left|t-x^{0}\right| \leq \bar{\Psi}}\left|f_{\varepsilon}^{(i)}(t)\right| \leq C_{x, i}, i=\overline{0, q}
\end{gathered}
$$

and for every $x, y \in \mathbb{R}^{1}$,

$$
\left|f_{\varepsilon}^{(q)}(x)-f_{\varepsilon}^{(q)}(y)\right| \leq L_{x}|x-y|^{\gamma_{x}}
$$

are fulfilled.

\subsubsection{Example of Noises $\Delta$}

Assume that noises $\left(\delta_{i}\right)$ satisfy the following autoregressive equation

$$
\delta_{i}=\lambda \delta_{i-1}+\eta_{i}, \quad i \geq 1,
$$

which is supposed to be stable $|\lambda|<1$, and $\eta_{i}$ are i.i.d., $E \eta_{i}=0, E \eta_{i}^{2} \leq \sigma_{\eta}^{2}, i \geq 0 ; \eta_{0}=\delta_{0}$. Then

$$
\varphi(k)=\frac{|\lambda|^{k} \sigma_{\eta}^{2}}{1-\lambda^{2}}
$$

In this case we can put (after optimization of the upper bound of the MSE)

$$
l_{n} \sim(1+\mu) \log _{|\lambda|} \frac{1}{n}, \quad \mu>0
$$

and the summands

$$
\frac{l_{n}}{n h_{n}} \sim \frac{\log n}{n h_{n}}, \quad \varphi_{n} h_{n}^{-1} \sim \frac{1}{n^{1+\mu} h_{n}}=o\left(\frac{1}{n h_{n}}\right) .
$$

Then the condition (3.4) (which is necessary for truncated estimators as well) is fulfilled if

$$
\frac{\log n}{n h_{n}}=o(1) \quad \text { as } n \rightarrow \infty .
$$

It is clear, that this example can be easy generalized for the stable autoregressive process (5.2) of an arbitrary order.

\subsection{Case of Dependent Inputs $X$ and $\Delta$}

Consider the estimation problem in the nonlinear autoregressive model

$$
Y_{i}=f\left(Y_{i-1}\right)+\delta_{i}, \quad i \geq 1,
$$

where $\delta_{i}, i \geq 1$ form the sequence of zero mean i.i..d.r.v's with a density function $f_{\delta}(\cdot)$ and finite variance $E \delta_{i}^{2}=\sigma^{2}, i \geq 1 ; Y_{0}$ is a zero mean random variable with finite variance and independent from $\delta_{i}, i \geq 1$.

Then $x_{n}=Y_{n-1}, \quad n \geq 0$ and $\varphi(k)=\sigma^{2} \chi(k=0), \quad k \geq 0$ and in this case $f_{i}(t)=f_{\delta}(t-$ $\left.\left.f\left(Y_{i-1}\right)\right) \mid \mathcal{F}_{i-1}^{Y}\right), \mathcal{F}_{i}^{Y}=\sigma\left\{Y_{0}, \delta_{1}, \ldots, \delta_{i}\right\}$. 
Thus Assumption (X) holds true if the function $f(\cdot)$ is uniformly bounded $\sup _{t \in \mathbb{R}^{1}} f(t) \leq C_{f}$, the density function $f_{\delta}(\cdot)$ is $q$-th differentiable, $q \geq 0$ and for some positive numbers $c_{x}^{0}, C_{x, 0}, L_{x}$ and $\gamma_{x} \in(0,1]$ the following relations

$$
\begin{gathered}
\inf _{\left|t-x^{0}\right| \leq C_{f}} f_{\delta}(t) \geq c_{x}^{0}, \\
\sup _{\left|t-x^{0}\right| \leq C_{f}} f_{\delta}^{(i)}(t) \leq C_{x, i}, i=\overline{0, q}
\end{gathered}
$$

and for every $x, y \in \mathbb{R}^{1}$,

$$
\left|f_{\delta}^{(q)}(x)-f_{\delta}^{(q)}(y)\right| \leq L_{x}|x-y|^{\gamma_{x}}
$$

are fulfilled.

\section{CONCLUSION}

The sequential approach for the problem of estimation of a regression function from dependent observations is developed. It is supposed, that the function to be estimated belongs to a Hölder class and input processes of the model can be unbounded with a positive probability.

Two types estimators are presented. Sequential estimators give the possibility to get estimators with an arbitrary mean square accuracy by finite stopping time. Truncated sequential estimators have known variance and constructed by a sample of finite (fixed) size.

Both estimation procedures work under general dependency types of model inputs. Asymptotic rate of convergence of both estimators coincides with the optimal rate of Nadaraya-Watson estimators constructed from independent observations. At that we consider the mean $E \tau_{n}$ having the rate (3.2) as a duration of observations in sequential approach for comparison with Nadaraya-Watson estimators calculated by the sample of the size $n$.

Presented estimators can be used directly and as pilot estimators in various statistical problems.

In this paper we have assumed that the function to be estimated has a scalar argument. In most applications, in particular to dynamic systems, the regressors will have a higher dimension. The extension to this case is immediate.

\section{APPENDIX}

\subsection{Proof of Theorem 3.1}

First we note that by the Definition 3.1 for the closeness of stopping times $\tau_{n}, n \geq 1$ it is enough to verify for every $n \geq n_{0}$ the following equalities

$$
\sum_{i=1}^{\infty} K\left(\frac{x^{0}-x_{i}}{h_{n}}\right)=+\infty \quad \text { a.s. }
$$

To this end we consider for every $n \geq n_{0}$ and $N \geq 1$ the following representation

$$
\begin{aligned}
& \frac{1}{N h_{n}} \sum_{i=1}^{N} K\left(\frac{x^{0}-x_{i}}{h_{n}}\right)=\frac{1}{N h_{n}} \sum_{i=1}^{N} E\left[K\left(\frac{x^{0}-x_{i}}{h_{n}}\right) \mid \mathcal{F}_{i-1}\right] \\
& +\frac{1}{N h_{n}} \sum_{i=1}^{N}\left(K\left(\frac{x^{0}-x_{i}}{h_{n}}\right)-E\left[K\left(\frac{x^{0}-x_{i}}{h_{n}}\right) \mid \mathcal{F}_{i-1}\right]\right) .
\end{aligned}
$$


Using the Assumptions (X) and (K), the first summand in the right hand side of this formula can be estimated from below

$$
\begin{gathered}
\frac{1}{N h_{n}} \sum_{i=1}^{N} E\left[K\left(\frac{x^{0}-x_{i}}{h_{n}}\right) \mid \mathcal{F}_{i-1}\right]=\frac{1}{N} \sum_{i=1}^{N} \int K(z) f_{i-1}\left(x^{0}-h_{n} z\right) d z \\
=\frac{1}{N} \sum_{i=1}^{N} f_{i-1}\left(x^{0}\right)+\frac{1}{N} \sum_{i=1}^{N} \int K(z)\left[f_{i-1}\left(x^{0}-h_{n} z\right)-f_{i-1}\left(x^{0}\right)\right] d z \\
=\frac{1}{N} \sum_{i=1}^{N} f_{i-1}\left(x^{0}\right)+\sum_{j=1}^{q} \frac{(-1)^{j}}{j !} \frac{h_{n}^{j}}{N} \sum_{i=1}^{N} f_{i-1}^{(j)}\left(x^{0}\right) \int z^{j} K(z) d z \\
+\frac{(-1)^{q}}{q !} \frac{h_{n}^{q}}{N} \sum_{i=1}^{N} \int z^{q} K(z)\left[f_{i-1}^{(q)}\left(x^{0}-\theta_{q, n, z} h_{n} z\right)-f_{i-1}^{(q)}\left(x^{0}\right)\right] d z \\
\geq c_{x}^{0}-\kappa h_{n}^{q+\gamma_{x}} \geq c_{x}^{0}-\kappa h_{n_{0}}^{q+\gamma_{x}}>0,
\end{gathered}
$$

where the number $\theta_{q, n, z} \in[0,1]$.

As regards to the second summand we note, that for every fixed $n \geq n_{0}$ according to Assumption (K) the processes $\left(M_{n, N}\right)_{N \geq 1}$,

$$
M_{n, N}=\sum_{i=1}^{N} \frac{1}{i}\left\{K\left(\frac{x^{0}-x_{i}}{h_{n}}\right)-E\left[K\left(\frac{x^{0}-x_{i}}{h_{n}}\right) \mid \mathcal{F}_{i-1}\right]\right\}
$$

form square integrable martingales with uniformly bounded variances:

$$
\sup _{N \geq 1} E M_{n, N}^{2}<\infty, \quad n \geq 1 .
$$

Thus, from the Doob theorem for square integrable martingales, see Liptser and Shiryaev (1988) and the Kronecker lemma (see, e.g., Loeve (1963)), the second summand goes to zero a.s.

Therefore the relations (7.1) and the finiteness of the stopping times $\tau_{n}$ are proven.

For the proof of the first assertion of Theorem 3.1 we note that the stopping times $\tau_{n}, n \geq 1$ are $\left\{\mathcal{F}_{i-1}\right\}$ adaptive, i.e. $\left\{i \leq \tau_{n}\right\} \in \mathcal{F}_{i-1}, i>1, n \geq 1$. Thus, by the definition of $\tau_{n}$ and using a well-known technique of sequential analysis (see, e.g., Borisov and Konev (1977); Dobrovidov at al. (2012); Konev (1985); Küchler and Vasiliev (2010); Liptser and Shiryaev (1978); Wald (1947, 1950) among others) and Assumption (X), we have

$$
\begin{gathered}
H_{n}>E \sum_{i=1}^{\tau_{n}-1} K\left(\frac{x^{0}-x_{i}}{h_{n}}\right)=E \sum_{i=1}^{\tau_{n}} K\left(\frac{x^{0}-x_{i}}{h_{n}}\right)-E K\left(\frac{x^{0}-x_{\tau_{n}}}{h_{n}}\right) \\
\geq E \sum_{i=1}^{\tau_{n}} E\left[K\left(\frac{x^{0}-x_{i}}{h_{n}}\right) \mid \mathcal{F}_{i-1}\right]-\bar{K}=h_{n} E \sum_{i=1}^{\tau_{n}} \int K(z) f_{i-1}\left(x^{0}-h_{n} z\right) d z-\bar{K} \\
=h_{n} E \sum_{i=1}^{\tau_{n}} f_{i-1}\left(x^{0}\right)+h_{n} E \sum_{i=1}^{\tau_{n}} \int K(z)\left(f_{i-1}\left(x^{0}-h_{n} z\right)-f_{i-1}\left(x^{0}\right)\right) d z-\bar{K} \\
=h_{n} E \sum_{i=1}^{\tau_{n}} f_{i-1}\left(x^{0}\right)+\sum_{j=1}^{q} \frac{(-1)^{j}}{j !} \frac{h_{n}^{j+1}}{H_{n}} E \sum_{i=1}^{\tau_{n}} f_{i-1}^{(j)}\left(x^{0}\right) \int z^{j} K(z) d z
\end{gathered}
$$




$$
\begin{gathered}
+\frac{(-1)^{q}}{q !} h_{n}^{q+1} E \sum_{i=1}^{\tau_{n}} \int z^{m} K(z)\left[f_{i-1}^{(q)}\left(x^{0}-\theta_{q, n, z} h_{n} z\right)-f_{i-1}^{(q)}\left(x^{0}\right)\right] d z \\
\geq c_{x}^{0} h_{n} E \tau_{n}-\kappa h_{n}^{q+1+\gamma_{x}} E \tau_{n}-\bar{K}
\end{gathered}
$$

Then

$$
E \tau_{n} \leq\left(c_{x}^{0}-\kappa h_{n}^{q+\gamma_{x}}\right)^{-1} h_{n}^{-1}\left(H_{n}+\bar{K}\right), \quad n \geq n_{0}
$$

On the other hand

$$
\begin{gathered}
H_{n} \leq E \sum_{i=1}^{\tau_{n}} K\left(\frac{x^{0}-x_{i}}{h_{n}}\right)=h_{n} E \sum_{i=1}^{\tau_{n}} \int K(z) f_{i-1}\left(x^{0}-h_{n} z\right) d z \\
=h_{n} E \sum_{i=1}^{\tau_{n}} f_{i-1}\left(x^{0}\right)+h_{n} E \sum_{i=1}^{\tau_{n}} \int K(z)\left(f_{i-1}\left(x^{0}-h_{n} z\right)-f_{i-1}\left(x^{0}\right)\right) d z \\
\leq h_{n}\left(C_{x, 0}+\kappa h_{n}^{q+\gamma_{x}}\right) E \tau_{n}
\end{gathered}
$$

and as follows

$$
E \tau_{n} \geq\left(C_{x, 0}+\kappa h_{n}^{q+\gamma_{x}}\right)^{-1} h_{n}^{-1} H_{n}, \quad n \geq 1 .
$$

Similarly, we have

$$
\begin{gathered}
H_{n}^{2}>E\left[\sum_{i=1}^{\tau_{n}-1} K\left(\frac{x^{0}-x_{i}}{h_{n}}\right)\right]^{2} \geq E\left[\sum_{i=1}^{\tau_{n}} K\left(\frac{x^{0}-x_{i}}{h_{n}}\right)\right]^{2} \\
-2 \bar{K} E \sum_{i=1}^{\tau_{n}} K\left(\frac{x^{0}-x_{i}}{h_{n}}\right) \geq E\left\{\sum_{i=1}^{\tau_{n}} E\left[K\left(\frac{x^{0}-x_{i}}{h_{n}}\right) \mid \mathcal{F}_{i-1}\right]\right\}^{2} \\
-2\left(C_{x, 0}+\kappa h_{n}^{q+\gamma_{x}}\right) h_{n} \sqrt{E \tau_{n}^{2}} \cdot \sqrt{E \sum_{i=1}^{\tau_{n}}\left(K\left(\frac{x^{0}-x_{i}}{h_{n}}\right)-E\left[K\left(\frac{x^{0}-x_{i}}{h_{n}}\right) \mid \mathcal{F}_{i-1}\right]\right)^{2}} \\
-2 \bar{K} h_{n} \sum_{i=1}^{\tau_{n}} K(y) f_{i-1}\left(x^{0}-h_{n} y\right) d y \geq\left(c_{x}^{0}-\kappa h_{n}^{q+\gamma_{x}}\right)^{2} h_{n}^{2} E \tau_{n}^{2}-2\left(C_{x, 0}+\kappa h_{n}^{q+\gamma_{x}}\right) h_{n} \sqrt{E \tau_{n}^{2}} \\
\times \sqrt{h_{n} E \sum_{i=1}^{\tau_{n}} \int\left[K(y)-E\left[K\left(\frac{x^{0}-x_{i}}{h_{n}}\right) \mid \mathcal{F}_{i-1}\right]\right]^{2} f_{i-1}\left(x^{0}-h_{n} y\right) d y-2 \bar{K} K_{0} C_{x, 0} h_{n} E \tau_{n}} \\
\geq\left(c_{x}^{0}-\kappa h_{n}^{q+\gamma_{x}}\right)^{2} h_{n}^{2} E \tau_{n}^{2}-2\left(C_{x, 0}+\kappa h_{n}^{q+\gamma_{x}}\right) h_{n} \sqrt{E \tau_{n}^{2}} \cdot \sqrt{C_{3} h_{n} E \tau_{n}} \\
-2 \bar{K} K_{0} C_{x, 0} h_{n}\left(c_{x}^{0}-\kappa h_{n}^{q+\gamma_{x}}\right)^{-1} h_{n}^{-1}\left(H_{n}+\bar{K}\right) \geq\left(c_{x}^{0}-\kappa h_{n}^{q+\gamma_{x}}\right)^{2} h_{n}^{2} \cdot E \tau_{n}^{2} \\
-2\left(C_{x, 0}+\kappa h_{n}^{q+\gamma_{x}}\right) h_{n} \cdot \sqrt{C_{3}\left(c_{x}^{0}-\kappa h_{n}^{q+\gamma_{x}}\right)^{-1}\left(H_{n}+\bar{K}\right) \cdot \sqrt{E \tau_{n}^{2}}} \\
-2 \bar{K} K_{0} C_{x, 0}\left(c_{x}^{0}-\kappa h_{n}^{q+\gamma_{x}}\right)^{-1}\left(H_{n}+\bar{K}\right) .
\end{gathered}
$$

Thus we have

$$
E \tau_{n}^{2} \leq\left\{\frac{\left(C_{x, 0}+\kappa h_{n}^{q+\gamma_{x}}\right) \cdot \sqrt{C_{3}\left(H_{n}+\bar{K}\right)}}{\left(c_{x}^{0}-\kappa h_{n}^{q+\gamma_{x}}\right)^{5 / 2} h_{n}}\right.
$$




$$
\left.+\frac{\sqrt{\left[C_{3}\left(C_{x, 0}+\kappa h_{n}^{q+\gamma_{x}}\right)^{2} \cdot\left(c_{x}^{0}-\kappa h_{n}^{q+\gamma_{x}}\right)^{-3}+2 \bar{K}^{2}\left(c_{x}^{0}-\kappa h_{n}^{q+\gamma_{x}}\right)^{-1}\right]\left(H_{n}+\bar{K}\right)+H_{n}^{2}}}{\left(c_{x}^{0}-\kappa h_{n}^{q+\gamma_{x}}\right) h_{n}}\right\}^{2} .
$$

Then the first assertion of the theorem follows from the definition of the function $t_{n}$. $f_{n}^{*}:$

Further, from Definition 3.1 and (1.1) we find the formula for the deviation of the sequential estimator

$$
f_{n}^{*}-f=I_{n}+J_{n}
$$

where

$$
I_{n}=\frac{1}{Q_{n}} \sum_{i=1}^{\tau_{n}}\left(f\left(x_{i}\right)-f\left(x^{0}\right)\right) K\left(\frac{x^{0}-x_{i}}{h_{n}}\right), \quad J_{n}=\frac{1}{Q_{n}} \sum_{i=1}^{\tau_{n}} \delta_{i} K\left(\frac{x^{0}-x_{i}}{h_{n}}\right) .
$$

Consider the bias of the estimator $f_{n}^{*}$ using the representation (7.2) for its deviation and a technique of conditional mathematical expectations.

$$
\begin{gathered}
E I_{n}=E \frac{1}{Q_{n}} \sum_{i=1}^{\tau_{n}}\left(f\left(x_{i}\right)-f\left(x^{0}\right)\right) K\left(\frac{x^{0}-x_{i}}{h_{n}}\right) \\
=E \frac{h_{n}}{Q_{n}} \sum_{i=1}^{\tau_{n}} \int K(z)\left(f\left(x^{0}-h_{n} z\right)-f\left(x^{0}\right)\right) f_{i-1}\left(x^{0}-h_{n} z\right) d z .
\end{gathered}
$$

By the Tailor expansion for the functions $f\left(x^{0}-h_{n} z\right)-f\left(x^{0}\right)$ and $f_{i-1}\left(x^{0}-h_{n} z\right)$ at a point $x^{0}$ up to the orders $p$ and $q$ respectively (Assumptions (f) and (X)) and according to Assumption (K) we have

$$
\begin{gathered}
\left|\int K(z)\left(f\left(x^{0}-h_{n} z\right)-f\left(x^{0}\right)\right) f_{i-1}\left(x^{0}-h_{n} z\right) d z\right|=\mid \frac{(-1)^{q}}{q !} h_{n}^{q} \\
\times \sum_{j=1}^{p} \frac{(-1)^{j}}{j !} h_{n}^{j} f^{(j)}\left(x^{0}\right) \int z^{q+j} K(z)\left(f_{i-1}^{(q)}\left(x^{0}-\theta_{q, n, z} h_{n} z\right)-f_{i-1}^{(q)}\left(x^{0}\right)\right) d z \\
+\frac{(-1)^{p}}{p !} h_{n}^{p} \int z^{p} K(z)\left(f^{(p)}\left(x^{0}-\tilde{\theta}_{q, n, z} h_{n} z\right)-f^{(p)}\left(x^{0}\right)\right) f_{i-1}\left(x^{0}-h_{n} z\right) d z \mid \\
\leq C_{1} h_{n}^{q+1+\gamma_{x}}+C_{2} h_{n}^{p+\gamma_{f}},
\end{gathered}
$$

where $\tilde{\theta}_{q, n, z} \in[0,1]$.

Thus, using the first assertion of the theorem, we obtain

$$
\begin{gathered}
\left|E I_{n}\right| \leq \frac{1}{H_{n}}\left[C_{1} h_{n}^{q+2+\gamma_{x}}+C_{2} h_{n}^{p+1+\gamma_{f}}\right] E \tau_{n} \\
\leq \frac{1}{H_{n}}\left[C_{1} h_{n}^{q+2+\gamma_{x}}+C_{2} h_{n}^{p+1+\gamma_{f}}\right]\left(c_{x}^{0}-\kappa h_{n}^{q+\gamma_{x}}\right)^{-1} h_{n}^{-1}\left(H_{n}+\bar{K}\right) \\
=\left(c_{x}^{0}-\kappa h_{n}^{q+\gamma_{x}}\right)^{-1}\left[C_{1} h_{n}^{q+1+\gamma_{x}}+C_{2} h_{n}^{p+\gamma_{f}}\right]\left(1+\frac{\bar{K}}{H_{n}}\right) .
\end{gathered}
$$

According to Assumptions (K) and $(\Delta)$ we have

$$
E J_{n}^{2} \cdot \chi_{\left\{\tau_{n} \leq l_{n}\right\}} \leq \gamma_{\delta} \frac{1}{H_{n}^{2}} \sum_{i=1}^{l_{n}} \sum_{j=1}^{l_{n}} E\left|K\left(\frac{x^{0}-x_{i}}{h_{n}}\right) K\left(\frac{x^{0}-x_{j}}{h_{n}}\right)\right| \cdot \varphi(|i-j|)
$$




$$
\leq \varphi(0) \bar{K}^{2} \gamma_{\delta} \frac{l_{n}}{H_{n}^{2}}\left(1+l_{n}\right)
$$

Further, by Definition 3.1 we can estimate

$$
\begin{gathered}
E J_{n}^{2} \cdot \chi_{\left\{\tau_{n}>l_{n}\right\}} \leq \frac{\varphi(0)}{H_{n}^{2}} E \sum_{i=1}^{\tau_{n}} K^{2}\left(\frac{x^{0}-x_{i}}{h_{n}}\right) \\
+\frac{2}{H_{n}^{2}} E \sum_{1 \leq i<j \leq \tau_{n}} \varphi(j-i) K\left(\frac{x^{0}-x_{i}}{h_{n}}\right) K\left(\frac{x^{0}-x_{j}}{h_{n}}\right) \cdot \chi_{\left\{\tau_{n}>l_{n}\right\}} \\
\leq \frac{\varphi(0)}{H_{n}^{2}} E \sum_{i=1}^{\tau_{n}} E\left[K^{2}\left(\frac{x^{0}-x_{i}}{h_{n}}\right) \mid \mathcal{F}_{i-1}^{x}\right]+\frac{2}{H_{n}^{2}} E S_{n} \leq \varphi(0) C_{3} \frac{h_{n} E \tau_{n}}{H_{n}^{2}}+\frac{2}{H_{n}^{2}} E S_{n},
\end{gathered}
$$

where

$$
S_{n}=\sum_{j=2}^{\tau_{n}} K\left(\frac{x^{0}-x_{j}}{h_{n}}\right) \sum_{i=1}^{j-1} K\left(\frac{x^{0}-x_{i}}{h_{n}}\right) \varphi(j-i) \cdot \chi_{\left\{\tau_{n}>l_{n}\right\}}
$$

We have

$$
\begin{gathered}
S_{n}=\sum_{j=2}^{l_{n}} K\left(\frac{x^{0}-x_{j}}{h_{n}}\right) \sum_{i=1}^{j-1} K\left(\frac{x^{0}-x_{i}}{h_{n}}\right) \varphi(j-i) \cdot \chi_{\left\{\tau_{n}>l_{n}\right\}} \\
+\sum_{j=l_{n}+1}^{\tau_{n}} K\left(\frac{x^{0}-x_{j}}{h_{n}}\right) \sum_{i=1}^{j-l_{n}} K\left(\frac{x^{0}-x_{i}}{h_{n}}\right) \varphi(j-i) \cdot \chi_{\left\{\tau_{n}>l_{n}\right\}} \\
+\sum_{j=l_{n}+1}^{\tau_{n}} K\left(\frac{x^{0}-x_{j}}{h_{n}}\right) \sum_{i=j-l_{n}+1}^{j-1} K\left(\frac{x^{0}-x_{i}}{h_{n}}\right) \varphi(j-i) \cdot \chi_{\left\{\tau_{n}>l_{n}\right\}} \\
\leq \gamma_{\delta} \varphi(0)\left[\sum_{j=1}^{l_{n}}\left|K\left(\frac{x^{0}-x_{j}}{h_{n}}\right)\right|\right]^{2}+\gamma_{\delta} \varphi_{n} \sum_{j=l_{n}+1}^{\tau_{n}}\left|K\left(\frac{x^{0}-x_{j}}{h_{n}}\right)\right| \sum_{i=1}^{j-l_{n}}\left|K\left(\frac{x^{0}-x_{i}}{h_{n}}\right)\right| \cdot \chi_{\left\{\tau_{n}>l_{n}\right\}} \\
+\gamma_{\delta} \varphi(0) \sum_{j=l_{n}+1}^{\tau_{n}}\left|K\left(\frac{x^{0}-x_{j}}{h_{n}}\right)\right| \sum_{i=j-l_{n}+1}^{j-1}\left|K\left(\frac{x^{0}-x_{i}}{h_{n}}\right)\right| \cdot \chi_{\left\{\tau_{n}>l_{n}\right\}} \\
\leq \gamma_{\delta} \varphi(0) l_{n}^{2} \bar{K}^{2}+\gamma_{\delta} K_{0} h_{n} \varphi_{n} E \tau_{n} \sum_{j=1}^{\tau_{n}}\left|K\left(\frac{x^{0}-x_{j}}{h_{n}}\right)\right|+\gamma_{\delta} \varphi(0) \bar{K} l_{n} \sum_{j=1}^{\tau_{n}}\left|K\left(\frac{x^{0}-x_{j}}{h_{n}}\right)\right| \\
\leq \gamma_{\delta} \varphi(0) l_{n}^{2} \bar{K}^{2}+\gamma_{\delta} K_{0} \bar{K} h_{n} \varphi_{n} E \tau_{n}^{2}+\gamma_{\delta} \varphi(0) K_{0} \bar{K} l_{n} h_{n} E \tau_{n} .
\end{gathered}
$$

Then, by the definition of $w_{n}$ and from (3.2), (3.3) and (7.5)-(7.7) it follows

$$
\begin{gathered}
E J_{n}^{2} \leq \varphi(0) \bar{K}^{2} \gamma_{\delta} \frac{l_{n}}{H_{n}^{2}}\left(1+3 l_{n}\right)+C_{3} \varphi(0) \frac{h_{n} E \tau_{n}}{H_{n}^{2}} \\
+2 \gamma_{\delta} \varphi(0) K_{0} \bar{K} \frac{l_{n} h_{n} E \tau_{n}}{H_{n}^{2}}+2 \gamma_{\delta} K_{0} \bar{K} \varphi_{n} \frac{h_{n} E \tau_{n}^{2}}{H_{n}^{2}} \leq w_{n}^{2}
\end{gathered}
$$

and, by the definition of $b_{n}$ as well as (7.4), (7.8) we have

$$
\left|E f_{n}^{*}-f\right| \leq\left|E I_{n}\right|+\sqrt{E J_{n}^{2}} \leq b_{n}
$$


The second assertion of Theorem 3.1 is proved.

For the proof of the third assertion we define the function

$$
\begin{aligned}
& \bar{f}_{n}^{*}=\frac{1}{Q_{n}} \sum_{i=1}^{\tau_{n}} E\left[K\left(\frac{x^{0}-x_{i}}{h_{n}}\right) f\left(x_{i}\right) \mid \mathcal{F}_{i-1}\right] \\
= & \frac{h_{n}}{Q_{n}} \sum_{i=1}^{\tau_{n}} \int K(z) f\left(x^{0}-h_{n} z\right) f_{i-1}\left(x^{0}-h_{n} z\right) d z .
\end{aligned}
$$

Now we estimate the second moment

$$
\begin{gathered}
E\left(f_{n}^{*}-\bar{f}_{n}^{*}\right)^{2} \leq\left(1+\gamma_{x, \delta}\right)\left[E \frac { 1 } { Q _ { n } ^ { 2 } } \left(\sum _ { i = 1 } ^ { \tau _ { n } } \left[f\left(x_{i}\right) K\left(\frac{x^{0}-x_{i}}{h_{n}}\right)\right.\right.\right. \\
\left.\left.\left.-E\left[K\left(\frac{x^{0}-x_{i}}{h_{n}}\right) f\left(x_{i}\right) \mid \mathcal{F}_{i-1}\right]\right]\right)^{2}+E J_{n}^{2}\right] \\
\leq\left(1+\gamma_{x, \delta}\right)\left[\frac{1}{H_{n}^{2}} E \sum_{i=1}^{\tau_{n}}\left[f\left(x_{i}\right) K\left(\frac{x^{0}-x_{i}}{h_{n}}\right)-E\left[K\left(\frac{x^{0}-x_{i}}{h_{n}}\right) f\left(x_{i}\right) \mid \mathcal{F}_{i-1}\right]\right]^{2}+E J_{n}^{2}\right] \\
=\left(1+\gamma_{x, \delta}\right)\left[\frac { h _ { n } } { H _ { n } ^ { 2 } } E \sum _ { i = 1 } ^ { \tau _ { n } } \int \left[K(y) f\left(x^{0}-h_{n} y\right)\right.\right. \\
\left.\left.-E\left[K\left(\frac{x^{0}-x_{i}}{h_{n}}\right) f\left(x_{i}\right) \mid \mathcal{F}_{i-1}\right]\right]^{2} f_{i-1}\left(x^{0}-h_{n} y\right) d y+E J_{n}^{2}\right] \\
\leq\left(1+\gamma_{x, \delta}\right) \frac{4 h_{n}}{H_{n}^{2}} E \sum_{i=1}^{\tau_{n}} \int K^{2}(y) f^{2}\left(x^{0}-h_{n} y\right) f_{i-1}\left(x^{0}-h_{n} y\right) d y+w_{n}^{2} .
\end{gathered}
$$

Thus, using Tailor expansion for the functions $f\left(x^{0}-h_{n} y\right), f_{i-1}\left(x^{0}-h_{n} y\right)$ at a point $x^{0}$ we estimate

$$
\begin{gathered}
f^{2}\left(x^{0}-h_{n} y\right) \leq(p+2) \sum_{k=0}^{p+1} \frac{1}{(k !)^{2}} C_{f, k}^{2} y^{2 k+2\left(\gamma_{f}-1\right) \chi(k=p+1)}, \\
\left|f_{i-1}\left(x^{0}-h_{n} y\right)\right| \leq \sum_{j=0}^{q+1} \frac{1}{j !} C_{x, j}|y|^{j+\left(\gamma_{x}-1\right) \chi(j=q+1)}
\end{gathered}
$$

and by the definition of $C_{4}$ we have

$$
\begin{gathered}
E\left(f_{n}^{*}-\bar{f}_{n}^{*}\right)^{2} \leq\left(1+\gamma_{x, \delta}\right)\left[C_{4} \frac{h_{n}}{H_{n}^{2}} E \tau_{n}+w_{n}^{2}\right] \\
\leq\left(1+\gamma_{x, \delta}\right)\left[\frac{C_{4}}{H_{n}}\left(c_{x}^{0}-\kappa h_{n}^{q+\gamma_{x}}\right)^{-1}\left(1+\frac{\bar{K}}{H_{n}}\right)+w_{n}^{2}\right] .
\end{gathered}
$$

Consider the difference

$$
\bar{f}_{n}^{*}-f=\frac{h_{n}}{Q_{n}} \sum_{i=1}^{\tau_{n}} \int K(z)\left(f\left(x^{0}-h_{n} z\right)-f\left(x^{0}\right)\right) f_{i-1}\left(x^{0}-h_{n} z\right) d z
$$




$$
+f \frac{1}{Q_{n}} \sum_{i=1}^{\tau_{n}}\left\{E\left[K\left(\frac{x^{0}-x_{i}}{h_{n}}\right) \mid \mathcal{F}_{i-1}\right]-K\left(\frac{x^{0}-x_{i}}{h_{n}}\right)\right\} .
$$

Using (7.3), we have

$$
\begin{aligned}
& E\left(\bar{f}_{n}^{*}-f\right)^{2} \leq 2 E\left(\frac{h_{n}}{Q_{n}} \sum_{i=1}^{\tau_{n}} \int K(z)\left(f\left(x^{0}-h_{n} z\right)-f\left(x^{0}\right)\right) f_{i-1}\left(x^{0}-h_{n} z\right) d z\right)^{2} \\
& +2 E\left(f \frac{1}{Q_{n}} \sum_{i=1}^{\tau_{n}}\left\{E\left[K\left(\frac{x^{0}-x_{i}}{h_{n}}\right) \mid \mathcal{F}_{i-1}\right]-K\left(\frac{x^{0}-x_{i}}{h_{n}}\right)\right\}\right)^{2} \\
& \leq \frac{2 h_{n}^{2}\left(C_{1} h_{n}^{q+1+\gamma_{x}}+C_{2} h_{n}^{p+\gamma_{f}}\right)^{2}}{H_{n}^{2}} E \tau_{n}^{2} \\
& +\frac{2 C_{f, 0}^{2}}{H_{n}^{2}} E \sum_{i=1}^{\tau_{n}}\left\{E\left[K\left(\frac{x^{0}-x_{i}}{h_{n}}\right) \mid \mathcal{F}_{i-1}\right]-K\left(\frac{x^{0}-x_{i}}{h_{n}}\right)\right\}^{2} \\
& \leq \frac{2\left(C_{1} h_{n}^{q+1+\gamma_{x}}+C_{2} h_{n}^{p+\gamma_{f}}\right)^{2} h_{n}^{2} t_{n}^{2}}{H_{n}^{2}}+\frac{2 C_{3} C_{f, 0}^{2} h_{n}}{H_{n}^{2}} E \tau_{n} \\
& \leq \frac{2\left(C_{1} h_{n}^{q+1+\gamma_{x}}+C_{2} h_{n}^{p+\gamma_{f}}\right)^{2} h_{n}^{2} t_{n}^{2}}{H_{n}^{2}}+\frac{2 C_{3} C_{f, 0}^{2}}{H_{n}}\left(c_{x}^{0}-\kappa h_{n}^{q+\gamma_{x}}\right)^{-1}\left(1+\frac{\bar{K}}{H_{n}}\right) .
\end{aligned}
$$

Then the third assertion of the theorem follows from the definition of the function $V_{n},(7.9),(7.10)$ and the inequality

$$
E\left(f_{n}^{*}-f\right)^{2} \leq 2\left[E\left(f_{n}^{*}-\bar{f}_{n}^{*}\right)^{2}+E\left(\bar{f}_{n}^{*}-f\right)^{2}\right] \leq V_{n} .
$$

Thus Theorem 3.1 is proved.

\subsection{Proof of Theorem 4.1}

Consider the deviation of the estimator (4.1):

$$
\begin{gathered}
f_{N}^{*}(h, H)-f=\left[\hat{f}_{N}(h, H)-f\right] \cdot \chi\left(\sum_{i=1}^{N} K\left(\frac{x^{0}-x_{i}}{h}\right) \geq H\right) \\
+\left[\frac{C_{f, 0}}{2}-f\right] \cdot \chi\left(\sum_{i=1}^{N} K\left(\frac{x^{0}-x_{i}}{h}\right)<H\right) .
\end{gathered}
$$

Then

$$
\begin{gathered}
E\left(f_{N}^{*}(h, H)-f\right)^{2}=E\left[\hat{f}_{N}(h, H)-f\right]^{2} \cdot \chi\left(\sum_{i=1}^{N} K\left(\frac{x^{0}-x_{i}}{h}\right) \geq H\right) \\
+\left[\frac{C_{f, 0}}{2}-f\right]^{2} \cdot P\left(\sum_{i=1}^{N} K\left(\frac{x^{0}-x_{i}}{h}\right)<H\right) \leq \frac{1}{H^{2}} E\left[\sum_{i=1}^{\tau_{N}(h, H)}\left(Y_{i} K\left(\frac{x^{0}-x_{i}}{h_{n}}\right)-f\right)\right]^{2} \\
+\frac{C_{f, 0}^{2}}{4} \cdot P\left(\sum_{i=1}^{N} K\left(\frac{x^{0}-x_{i}}{h}\right)<H\right) .
\end{gathered}
$$


Similar to the proof of Theorem 3.1, using the inequalities $E \tau_{N}(h, H) \leq N$ and $E \tau^{2}(N, H) \leq N^{2}$ in (7.8), (7.9) and (7.10) instead of (3.2) and (3.3) for $E \tau_{n}$ and $E \tau_{n}^{2}$ can be proved the inequality:

$$
\begin{gathered}
\frac{1}{H^{2}} E\left[\sum_{i=1}^{\tau_{N}(h, H)}\left(Y_{i} K\left(\frac{x^{0}-x_{i}}{h}\right)-f\right)\right]^{2} \\
\leq 2\left\{\left(2 C_{3} C_{f, 0}^{2}+C_{4}\left(1+\gamma_{x, \delta}\right) \frac{h N}{H^{2}}+\left(1+\gamma_{x, \delta}\right)\left[\varphi(0) \bar{K}^{2} \gamma_{\delta} \frac{l(H)}{H^{2}}(1+3 l(H))\right.\right.\right. \\
\left.\left.+C_{3} \varphi(0) \frac{h N}{H^{2}}+2 \gamma_{\delta} \varphi(0) K_{0} \frac{l(H) h N}{H^{2}}+2 \gamma_{\delta} K_{0} \bar{K} \varphi(l(H)) \frac{h N^{2}}{H^{2}}\right]+\frac{2\left(C_{1} h^{q+1+\gamma_{x}}+C_{2} h^{p+\gamma_{f}}\right)^{2} h^{2} N^{2}}{H^{2}}\right\} .
\end{gathered}
$$

Now, using the Chebyshev inequality, for $N h\left(c_{x}^{0}-\kappa h^{q+\gamma_{x}}\right)-H>0$ we estimate the probability

$$
\begin{gathered}
P\left(\sum_{i=1}^{N} K\left(\frac{x^{0}-x_{i}}{h}\right)<H\right) \\
=P\left(\sum_{i=1}^{N}\left(E K\left[\left(\frac{x^{0}-x_{i}}{h}\right) \mid \mathcal{F}_{i-1}\right]-K\left(\frac{x^{0}-x_{i}}{h}\right)\right)>\sum_{i=1}^{N} E K\left[\left(\frac{x^{0}-x_{i}}{h}\right) \mid \mathcal{F}_{i-1}\right]-H\right) \\
\leq P\left(\sum_{i=1}^{N}\left(E K\left[\left(\frac{x^{0}-x_{i}}{h}\right) \mid \mathcal{F}_{i-1}\right]-K\left(\frac{x^{0}-x_{i}}{h}\right)\right)>N h\left(c_{x}^{0}-\kappa h^{q+\gamma_{x}}\right)-H\right) \\
\leq \frac{1}{\left(N h\left(c_{x}^{0}-\kappa h^{q+\gamma_{x}}\right)-H\right)^{2}} E\left(\sum_{i=1}^{N}\left(E K\left[\left(\frac{x^{0}-x_{i}}{h}\right) \mid \mathcal{F}_{i-1}\right]-K\left(\frac{x^{0}-x_{i}}{h}\right)\right)\right)^{2} \\
\leq \frac{C_{3} N h}{\left(N h\left(c_{x}^{0}-\kappa h^{q+\gamma_{x}}\right)-H\right)^{2}}
\end{gathered}
$$

and two first assertions of Theorem 4.1 are proved.

For the proof of the third assertion it is enough to prove the following limit relation:

$$
\lim _{N \rightarrow \infty} \frac{1}{H_{N}} \sum_{i=1}^{N} K\left(\frac{x^{0}-x_{i}}{h_{N}}\right)>1 \text { a.s. }
$$

To this end we note that, similarly to the beginning of the proof of Theorem 3.1 we have the inequalities

$$
\frac{1}{N h_{N}} \sum_{i=1}^{N} E\left[K\left(\frac{x^{0}-x_{i}}{h_{N}}\right) \mid \mathcal{F}_{i-1}\right] \geq c_{x}^{0}-\kappa h_{n_{0}}^{q+\gamma_{x}}>0, \quad N \geq n_{0} .
$$

Then we prove the following relations

$$
\begin{gathered}
E\left|\frac{1}{N h_{N}} \sum_{i=1}^{N} K_{i, N}\right|^{r} \leq \frac{\left(8 \bar{K}^{2} r\right)^{r / 2}}{N^{r / 2} h_{N}^{r-1}}, \quad N \geq 1, \\
K_{i, N}=K\left(\frac{x^{0}-x_{i}}{h_{N}}\right)-K\left[\left(\frac{x^{0}-x_{i}}{h_{N}}\right) \mid \mathcal{F}_{i-1}\right],
\end{gathered}
$$


using the covariance (Burkholder) inequality

$$
E\left|\sum_{i=1}^{N} X_{i}\right|^{m} \leq(2 m)^{m / 2}\left(\left(E\left(\left|X_{i}\right| \sum_{j=i}^{N}\left|E\left(X_{j} \mid \mathcal{F}_{i}\right)\right|\right)^{m / 2}\right)^{2 / m}\right)^{m / 2},
$$

which holds true, according to Dedecker and Doukhan (1990), for $m \geq 2$ and every sequence $\left(X_{i}, \mathcal{F}_{i}\right)_{1 \leq i \leq N}$, such that $\max _{1 \leq i \leq N} E\left|X_{i}\right|^{m}<\infty$.

Indeed, these inequalities are fulfilled for $m=r$ and $X_{i}=K_{i, N}$ in our case in view of the following relations:

$$
E\left(K_{j, N} \mid \mathcal{F}_{i}\right)=K_{j, N} \cdot \chi(j=i), \quad E\left[\left|K\left(\frac{x^{0}-x_{i}}{h_{N}}\right)\right| s \mid \mathcal{F}_{i-1}\right] \leq \bar{K}^{s} h_{N}, \quad s>0
$$

$C_{r}$-inequality: $|a+b| \leq C_{r}\left(|a|^{r}+|b|^{r}\right), C_{r}=1$ if $0<r<1 ; C_{r}=2^{r-1}$ if $r \geq 1$ and

$$
\begin{gathered}
E\left|K_{j, N}\right|^{r} \leq 2^{r-1} E\left(\left|K\left(\frac{x^{0}-x_{i}}{h_{N}}\right)\right|^{r}+\left|K\left[\left(\frac{x^{0}-x_{i}}{h_{N}}\right) \mid \mathcal{F}_{i-1}\right]\right|^{r}\right) \\
\leq 2^{r-1} E\left(E\left[\left|K\left(\frac{x^{0}-x_{i}}{h_{N}}\right)\right|^{r} \mid \mathcal{F}_{i-1}\right]+\left|K\left[\left(\frac{x^{0}-x_{i}}{h_{N}}\right) \mid \mathcal{F}_{i-1}\right]\right|^{r}\right) \leq(2 \bar{K})^{r} h_{N} .
\end{gathered}
$$

Thus, from the Borel-Cantelli lemma, condition from the third assertion of Theorem 4.1 and (7.14) we have

$$
\lim _{N \rightarrow \infty} \frac{1}{N h_{N}} \sum_{i=1}^{N} K_{i, N}=0 \quad \text { a.s. }
$$

and, using (7.13), we obtain

$$
\begin{gathered}
\lim _{N \rightarrow \infty} \frac{1}{H_{N}} \sum_{i=1}^{N} K\left(\frac{x^{0}-x_{i}}{h_{N}}\right)=\lim _{N \rightarrow \infty} \frac{C_{\alpha}}{N h_{N}} \sum_{i=1}^{N} E\left[K\left(\frac{x^{0}-x_{i}}{h_{N}}\right) \mid \mathcal{F}_{i-1}\right] \\
\geq \frac{c_{x}^{0}-\kappa h_{n_{0}}^{q+\gamma_{x}}}{c_{x}^{0}-\kappa h_{n_{0}}^{q+\gamma_{x}}-\alpha^{-1 / 2}}>1 \quad \text { a.s. }
\end{gathered}
$$

Theorem 4.1 is proved.

\section{REFERENCES}

Bai, Er-Wei, and Liu, Yun. (2007). Recursive Direct Weight Optimization in Nonlinear System Identification: A Minimal Probability Approach, IEEE Transactions on Automatic Control (July 2007) 52(7): $1218-1231$.

Borisov, V.Z., and Konev, V.V. (1977). On Sequential Estimation of Parameters in Discrete-time Processes, Automat. and Remote Control 10: 58-64 (in Russian).

Carroll, R.J. (1976). On Sequential Density Estimation, Zeitschrift für Warscheinlichskeitstheorie und Verwandte Geiete 36: 58-64.

Davies, H.I., and Wegman, E.J. (1975). Sequential Nonparametric Density Estimation, IEEE Transactions in Information Theory 21: 619-628.

Dedecker, J., and Doukhan, P. (1990). A New Covariance Inequality and Applications, Stochastic Proc. Appl. 10: 63-80. 
Dharmasena, S., Zeephongsekul, P., and De Silva. (2008). Two Stage Sequential Procedure for Nonparametric Regression Estimation, Australian and New Zealand Industrial and Applied Mathematics Journal 49: 699-716.

Dobrovidov, A.V., Koshkin, G.M., and Vasiliev, V.A. (2012). Non-parametric State Space Models, USA: Kendrick Press.

(Russian original: Dobrovidov, A.V., Koshkin, G.M., and Vasiliev, V.A. (2004). Nonparametric Estimation of Functionals of Stationary Sequences Distributions, Moscow.: Nauka.)

Efroimovich, S.Yu. (1999). Nonparametric Curve Estimation. Methods, Theory and Applications, Berlin, N. Y.: Springer-Verlag.

Efroimovich, Sam. (2007). Sequential Design and Estimation in Heteroscedastic Nonparametric Regression, Sequential Analysis: Design Methods and Applications 26(1): 3-25. DOI: 10.1080/07474940601109670

Györfi, L., Kohler, M., Krzyżak, A., and Walk, H.. (2002). A Distribution-free Theory of Nonparametric Regression, Berlin, N. Y.: Springer-Verlag.

Honda, T. (1998). Sequential Estimation of the Hgarginal Density Function for a Strongly Mixing Process, Sequential Analysis: Design Methods and Applications 17(3,4): 239-251. DOI: 10.1080/07474949808836411

Konev, V.V. (1985). Sequential Parameter Estimation of Stochastic Dynamical Systems, Tomsk: Tomsk Univ. Press (in Russian).

Konev, V.V., and Pergamenshchikov, S.M. (1990). Truncated Sequential Estimation of the Parameters in Random Regression, Sequential analysis 9(1): 19-41.

Konev, V.V., and Pergamenshchikov, S.M. (1990). On Truncated Sequential Estimation of the Drifting Parameter Mean in the First Order Autoregressive Model, Sequential analysis 9(2): 193-216.

Konev, V.V., and Pergamenshchikov, S. M. (1992). On Truncated Sequential Estimation of the Parameters of Diffusion Processes, Methods of Economical Analysis, Ed.: Central Economical and Mathematical Institute of Russian Academy of Science, Moscow 3-31.

Küchler, U., and Vasiliev, V. (2010). On Guaranteed Parameter Estimation of a Multiparameter Linear Regression Process, Automatica, Journal of IFAC, Elsevier 46: 637-646.

Fourdrinier, D., Konev, V., and Pergamenshchikov, S. (2009). Truncated Sequential Estimation of the Parameter of a First Order Autoregressive Process with Dependent Noises, Mathematical Methods of Statistics 18(1): 43-58.

Leonov, S.L. (1999). Remarks on Extremal Problems in Nonparametric Curve Estimation, Statistics and Probability Letters 43(2): 169-178.

Liptser R.Sh., and Shiryaev A.N. (1978). Statistics of Random Processes. I: General Theory. N. Y.: SpringerVerlag, 1977. 2: Applications. N. Y.: Springer-Verlag, 1978.

Liptser, R., and Shiryaev, A. (1988). Theory of Martingales. Springer Verlag, Berlin, Heidelberg, New York.

Liski, E.P., Mandal, N.K., Shah, K.R., and Sinha, B.K. (2002). Topics in Optimal Design, N. Y.: SpringerVerlag, 2: Applications. N. Y.: Springer-Verlag.

Loeve, M. (1963). Probability Theory. 3-d. ed., N. Y.: D.Van Nostrand.

Martinsek, A.T. (1992). Using Stopping Rules to Bound the Mean Integrated Squared Error in Density Estimation, Annals of Statistics 20: 797-806.

McMurry, T., and Politis, D.N. (2004). Nonparametric Regression with Infinite Order Flat-top Kernels, $J$. Nonparam. Statist. 16(3-4): 549-562.

Mukhopadhyay, N., and Solanky, T.K.S. (1994). Multistage Selection and Ranking Procedures, New York: Marcel Dekker.

Nazin, A.V., and Ljung, L. (2002). Asymptotically Optimal SMoothing of Averaged LMS for Regression // Proceedengs of the 15th IFAC World Congress, Barcelona. 
Novikov, A.A. (1971). Sequential Estimation of the Parameters of the Diffusion Processes, Theory Probab. Appl. 16(2): 394-396 (in Russian).

Politis, D.N. (2001). On Nonparametric Function Estimation with Infinite-order Flat-top Kernels, Probability and Statistical Models with applications, Ch. Charalambides et al. (Eds.), Chapman and Hall/CRC, Boca Raton 469-483.

Politis, D.N., and Romano, J.P. (1993). On a Family of Smoothing Kernels of Infinite Order, Proceedings of the 25th Symposium on the Interface, San Diego, California, April 14-17, (M. Tarter and M. Lock, eds.), The Interface Foundation of North America 141-145.

Politis, D.N., and Romano, J.P. (1999). Multivariate Density Estimation with General Flat-top Kernels of Infinite Order, Journal of Multivariate Analysis 68: 1-25.

Politis, D., Romano, J., and Wolf, M. (1999). Subsumpling, Springer Verlag, Berlin, Heidelberg, New York.

Politis, D.N., and Vasiliev, V.A. (2012). Sequential kernel estimation of a multivariate regression function, Proceedings of the IX International Conference 'System Identification and Control Problems', SICPRO'12, Moscow, 30 January - 2 February, 2012, V. A. Tapeznikov Institute of Control Sciences 996-1009.

Prakasa Rao, B.L.S. (1983) Nonparametric Functional Estimation, New York: Academic Press.

Roll, J., Nazin, A., and Ljung, L. (2002). A Non-Asymptotic Approach to Local Modelling. The 41st IEEE CDC, Las Vegas, Nevada, USA, 10-13 Dec. (Regular paper.)

http://www.control.isy.liu.se/research/reports/2003/2482.pdf

Roll, J., Nazin, A., and Ljung, L. (2005). Non-linear System Identification Via DirectWeight Optimization, Automatica, IFAC Journal, Special Issue on Data-Based Modelling and System Identification 41(3): 475490.

http://www.control.isy.liu.se/research/reports/2005/2696.pdf

Rosenblatt, M. (1991). Stochastic Curve Estimation, NSF-CBMS Regional Conference Series, 3, Institute of Mathematical Statistics, Hayward.

Scott, D.W. (1992). Multivariate Density Estimation: Theory, Practice and Visualization, Wiley, New York.

Stute, W. (1983). Sequential Fixed-Width Confidence Intervals for a Nonparametric Density Function. Zeitschrift für Warscheinlichskeitstheorie und Verwandte Geiete 62: 113-123.

Vasiliev, V.A. (1997). On Identification of Dynamic Systems of Autoregressive Type, Automat. and Remote Control 58: 12, 106-118.

Vasiliev, V.A. (2011). On Adaptive Control Problems of Discrete-time Stochastic Systems, Proceedings of the 18th World Congress The Int. Fed. Autom. Contr., Milan, Italy, August 27 - September 2, 6 pages (to be published).

Vasiliev, V.A., and Konev, V.V. (1997). On Optimal Adaptive Control of a Linear Stochastic Process, Proc.15th IMACS World Congress on Scientific Computation, Modelling and Applied Mathematics, August 24-29, Berlin, Germany 5: 87-91.

Vasiliev, V.A., and Koshkin, G.M. (1998). Nonparametric Identification of Autoregression, Probability Theory and Its Applications 43: 577-588.

Wald, A. (1947). Sequential Analysis, N. Y.: Wiley.

Wald, A. (1950). Statistical Decision Functions, N. Y.: Wiley.

Xu, Y., and Martinsek, A.T. (1995) Sequential Confidence Bands for Densities, Annals of Statistics 23: 2218-2240. 